\title{
Clinical impact of atrial fibrillation in a nationwide cohort of hypertrophic cardiomyopathy patients
}

\author{
Hyun-Jung Lee ${ }^{1}$, Hyung-Kwan Kim ${ }^{1}$, Minkwan Kim ${ }^{1}$, Inki Moon ${ }^{1}$, Heesun Lee ${ }^{1,2}$, Bong-Seong Kim ${ }^{3}$, \\ Kyung-Do Han ${ }^{3}$, Jun-Bean Park ${ }^{1}$, In-Chang Hwang ${ }^{4}$, Yeonyee E. Yoon ${ }^{4}$, Yong-Jin Kim ${ }^{1}$, Goo-Yeong Cho ${ }^{4}$, \\ Steve R. Ommen
}

${ }^{1}$ Department of Internal Medicine, Seoul National University Hospital, Seoul, Korea; ${ }^{2}$ Department of Internal Medicine, Healthcare System Gangnam Center, Seoul National University Hospital, Seoul, Korea; ${ }^{3}$ Department of Statistics and Actuarial Science, Soongsil University, Seoul, Korea; ${ }^{4}$ Cardiovascular Center and Department of Internal Medicine, Seoul National University Bundang Hospital, Seongnam, Gyeonggi, Korea; ${ }^{5}$ Division of Cardiovascular Diseases, Mayo Clinic College of Medicine, Rochester, Minnesota, USA

Contributions: (I) Conception and design: HJ Lee, HK Kim; (II) Administrative support: HK Kim; (III) Provision of study materials or patients: HJ Lee, HK Kim; (IV) Collection and assembly of data: HJ Lee, KD Han, BS Kim; (V) Data analysis and interpretation: KD Han, BS Kim, HJ Lee, HK Kim; (VI) Manuscript writing: All authors; (VII) Final approval of manuscript: All authors.

Correspondence to: Hyung-Kwan Kim, MD, PhD. Professor/Director of Cardiac Diagnostic Test Unit, Cardiovascular Center, Department of Internal Medicine, Seoul National University Hospital, 101 Daehak-ro, Jongno-gu, Seoul, Korea. Email: cardiman73@gmail.com.

Background: The clinical implications of atrial fibrillation (AF) in hypertrophic cardiomyopathy (HCM) patients are incompletely characterized. We investigated the impact of AF on stroke and mortality, assessed the performance of the CHA2DS2-VASc score, and explored the predictors of stroke in HCM patients.

Methods: A nationwide cohort of HCM patients ( $n=8,349$, mean age 60.7) identified from 2010 to 2015 from the Korean National Health Insurance database were followed up for occurrence of ischemic stroke or all-cause death.

Results: During a mean follow-up of 2.5 years, the incidence rate of stroke was 2.69 and 5.87 , and mortality rate was 2.06 and 4.44 per 100 person-years in non-AF and AF patients, respectively. AF was independently associated with a $60 \%$ and $50 \%$ increased risk for stroke and all-cause mortality, respectively. The AF-associated increase in risk of stroke was more prominent in HCM patients with no or few risk factors. The CHA2DS2-VASc score showed poor discrimination of stroke risk in HCM patients with AF, mostly due to the high incidence of stroke in patients with scores of 0 or 1 . Traditional risk factors were not always associated with stroke in HCM patients with AF; age, heart failure, high blood pressure and GGT were the strongest predictors of stroke in this population. HCM patients without AF also showed increased incidence of stroke at CHA2DS2-VASc $\geq 1$.

Conclusions: AF was independently associated with increased risks for stroke and all-cause mortality in patients with HCM. The CHA2DS2-VASc score showed poor discrimination of stroke risk in HCM patients with AF.

Keywords: Hypertrophic cardiomyopathy (HCM); atrial fibrillation (AF); stroke; mortality

Submitted Feb 22, 2020. Accepted for publication Sep 06, 2020.

doi: $10.21037 /$ atm-20-1817

View this article at: http://dx.doi.org/10.21037/atm-20-1817 


\section{Introduction}

Atrial fibrillation (AF) is the most common arrhythmia in hypertrophic cardiomyopathy (HCM), and has been associated with significant functional decline, morbidity, especially stroke, and mortality (1-4). However, advances in management strategies have substantially improved the clinical course and survival of HCM patients (5). In particular, $\mathrm{AF}$ was reported to be no longer a major contributor to morbidity and mortality in HCM in the modern management era (6). Meanwhile, other studies of HCM populations spanning longer time periods reported significantly increased adverse events and death attributable to $\mathrm{AF}(3,4)$. In this regard, the clinical implications of $\mathrm{AF}$ in HCM patients in the contemporary management era are incompletely characterized. Furthermore, there is a lack of data on the clinical impact of AF in Asian HCM patients. Also, the prediction of stroke in HCM patients is not straightforward, as the $\mathrm{CHA}_{2} \mathrm{DS}_{2}$-VASc score does not appear to correlate well with thromboembolic risk in HCM patients with AF (6,7). Although current guidelines recommend anticoagulation in all HCM patients who develop $\mathrm{AF}$ without a threshold of $\mathrm{CHA}_{2} \mathrm{DS}_{2}$-VASc scores $(8,9)$, concrete evidence for this recommendation is lacking.

Here, we investigated the clinical impact of AF on stroke and mortality, explored the predictors of stroke, and assessed the performance of the $\mathrm{CHA}_{2} \mathrm{DS}_{2}$-VASc score in a nationwide cohort of HCM patients. We present the following article in accordance with the STROBE reporting checklist (available at http://dx.doi.org/10.21037/atm-20-1817).

\section{Methods}

\section{Study population}

This study used the National Health Insurance Service (NHIS) database, which provides mandatory coverage for all Korean citizens. The database contains information on demographics, claims data on inpatient and outpatient health care usage, diagnoses, prescriptions, treatments, as well as the routine health examination data, and death information data for the entire Korean population, and can be used for population-based studies. The de-identified data is available to researchers who apply online (nhiss.nhis.or.kr) after approval by the Institutional Review Board. The NHIS recommends enrollees to undergo regular standardized health examinations annually or biennially depending on occupation. According to statistics from the Korean Statistical Information Service, the inspection rate was
$76.1 \%$ in a total of $17,633,406$ subjects scheduled to take the 2015 health examinations. Standardized health examinations were performed in hospitals certified by the NHIS and subjected to regular quality control. Questionnaires, anthropometric measurements, blood and urine tests, chest $\mathrm{x}$-ray, and dental examinations were included in the health examinations. Blood samples including measurement of serum glucose and lipid levels were drawn after an overnight fast. Information on smoking and alcohol consumption, physical activity was obtained by questionnaires. Smoking status was checked as non-smoker, ex-smoker, and current smoker, and alcohol consumption was checked as none, mild ( $<30 \mathrm{~g} /$ day), and heavy drinker ( $\geq 30 \mathrm{~g} /$ day). Regular exercise was defined as performing moderate physical activity more than 30 minutes at least five times per week or strenuous physical activity more than 20 minutes at least three times per week. Income data was included in the qualification database, and low income was defined as lowest $20 \%$.

From this database, we identified patients with HCM from 1 January 2010 to the 31 December 2015 ( $n=16,886)$. We excluded patients without health examination results within \pm 1 year of study enrollment $(n=6,436)$ or with missing values in health examination $(\mathrm{n}=127)$. Patients with history of stroke prior to enrollment were washed out in order to detect new-onset stroke $(\mathrm{n}=1,974)$, as prior and new events were difficult to differentiate with diagnostic codes in the claims database. Hence, a total of 8,349 HCM patients were finally included (Figure 1). Patients were classified into those with $\mathrm{AF}$, and those without. The study cohort was followed up until 31 December 2016. Baseline characteristics were collected in the year of enrollment, and initial health examination results were assessed. The study was conducted in accordance with the Declaration of Helsinki (as revised in 2013). This study was exempt from review by the Seoul National University Hospital Institutional Review Board (E1806-016-949) due to the anonymized nature of the data and individual consent was waived.

\section{Definitions}

Diseases were defined using the International Statistical Classification of Diseases and Related Health Problems, Tenth Revision, Clinical Modification (ICD-10-CM), health care usage and medication, as in previous studies (10-12), and are detailed in Table S1.

HCM was defined by (I) claims for diagnostic codes (I42.1 or I42.2) with at least one admission or outpatient clinic visit, and (II) registration in the rare intractable 


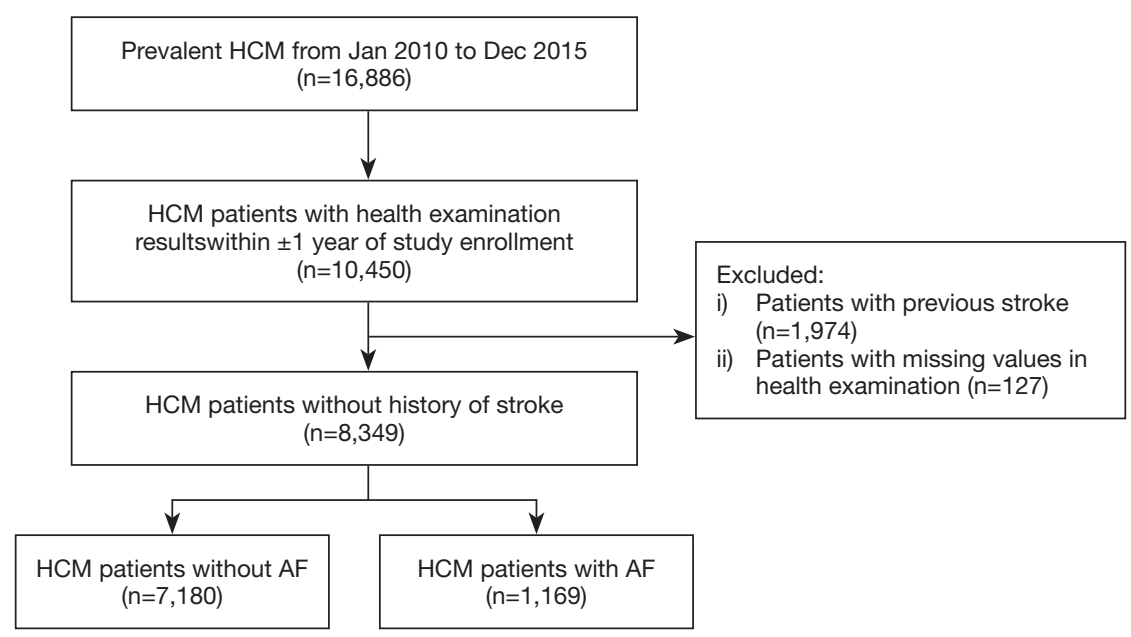

Figure 1 Study inclusion flow.

diseases (RID) program. The government-implemented RID program is a welfare policy extending health insurance coverage to $90 \%$ of medical costs for patients with specific rare diseases. Therefore, registration is tightly controlled by verification with clinical and imaging evidence, and reviews by medical experts and health insurance professionals. HCM was included in the rare diseases listed in the RID program since 2004. To be registered in the RID program, the patient must fulfill the criteria of HCM on echocardiography and the attending physician must provide clinical certification that the patient has HCM. When validated with hospital data $(n=1,110)$, the combination of ICD-10-CM codes and registration in the RID program showed a high positive predictive value and accuracy (13).

$\mathrm{AF}$ was defined with ICD-10-CM code I48, which was validated in previous studies $(10-12,14)$. $\mathrm{CHA}_{2} \mathrm{DS}_{2}$-VASc scores were calculated by assigning 2 points each for age $\geq 75$ years and prior stroke/transient ischemic attack/systemic thromboembolism and assigning 1 point each for age 65-74 years, female, congestive heart failure, hypertension, diabetes mellitus, and vascular disease (peripheral artery disease or prior myocardial infarction) (15). We used the $\mathrm{CHA}_{2} \mathrm{DS}_{2}$-VASc score to represent the number of risk factors for patients without $\mathrm{AF}$ as well, as this score has also been shown to correlate with stroke risk in patients without $\mathrm{AF}$ (16-18).

The endpoint was ischemic stroke and all-cause death. Ischemic stroke was defined by diagnosis with ICD-10-CM codes I63 or I64 during admission with claims for brain imaging. When validated with hospital data $(n=200)$, the positive predictive value for ischemic stroke was $94.3 \%$.

\section{Statistical analysis}

Data are presented as mean \pm standard deviation for continuous variables with normal distribution and proportions for categorical variables; triglycerides, alanine transaminase, aspartate transaminase (AST), gammaglutamyltransferase (GGT) levels showed positively skewed distributions and are presented as geometric means with $95 \%$ confidence intervals ( $95 \% \mathrm{CI}$ ) after logarithmic transformation. The Students' $t$-test and $\chi^{2}$ test were used to compare variables between groups.

Incidence rates were calculated per 100 person-years. Cox proportional hazards model was used to calculate hazard ratios (HR) and $95 \%$ CIs for the risk of stroke and death, and to identify independent predictors with multivariable regression analysis. Proportional hazards assumptions were tested graphically using log-log plots, and there was no significant departure from proportionality in hazards over time. The performances of the $\mathrm{CHA}_{2} \mathrm{DS}_{2}$-VASc score were evaluated in patients using receiver operating characteristic (ROC) curves with areas under the curves (AUCs) (19). Statistical analyses were performed using SAS version 9.4 (SAS Institute Inc., Cary, NC, USA), and 2-sided P values $<0.05$ were considered statistically significant.

\section{Results}

\section{Baseline characteristics of study population}

In a total of $8,349 \mathrm{HCM}$ patients (mean age $60.7 \pm 11.9$, male $69.2 \%)$, prevalence of AF was $14.0 \%(n=1,169)$. Patients with AF were significantly older $(64.6 \pm 10.8 v s .60 .1 \pm 12.0$ years $)$, 
had higher $\mathrm{CHA}_{2} \mathrm{DS}_{2}$-VASc scores $(2.8 \pm 1.6$ vs. $2.1 \pm 1.5)$, higher prevalence of most comorbidities including hypertension, heart failure, ischemic heart disease, peripheral artery disease, chronic obstructive pulmonary disease, and lower prevalence of dyslipidemia compared to non-AF counterparts (Table 1). They had lower systolic blood pressure, total cholesterol, low density lipoprotein-cholesterol, triglyceride, creatinine clearance and hemoglobin levels, and higher AST and GGT levels. Patients with AF were less likely to smoke, drink heavily, or exercise regularly. Of HCM patients with AF, approximately half $(51.3 \%)$ were on oral anticoagulants, mostly warfarin, while a very small proportion of HCM patients without AF $(1.6 \%)$ were on oral anticoagulants.

Table 1 Baseline characteristics of hypertrophic cardiomyopathy patients with and without atrial fibrillation

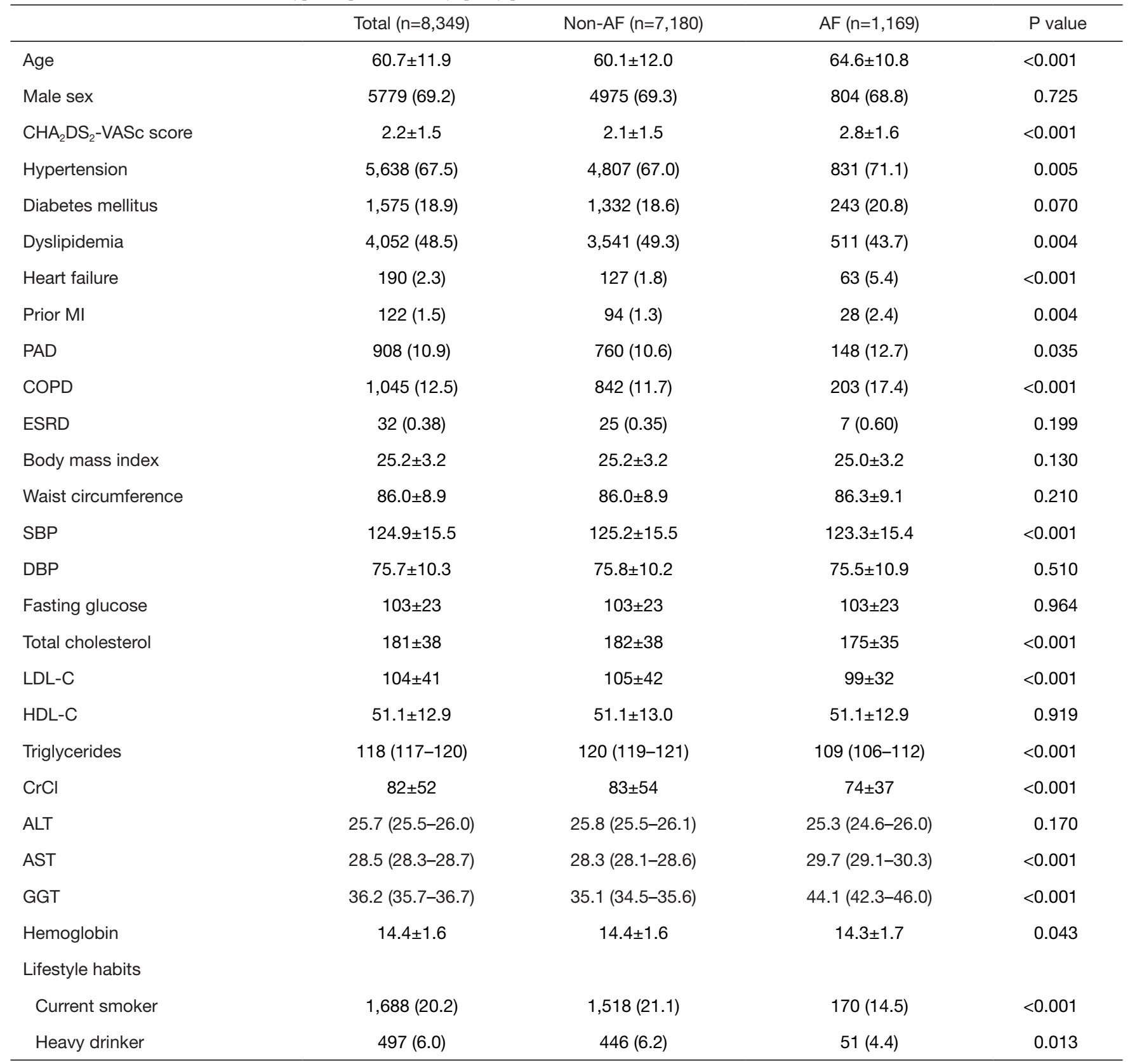

Table 1 (continued) 
Table 1 (continued)

\begin{tabular}{|c|c|c|c|c|}
\hline & Total $(\mathrm{n}=8,349)$ & Non-AF $(n=7,180)$ & $A F(n=1,169)$ & $P$ value \\
\hline Low income & $1,476(17.7)$ & 1,261 (17.6) & $215(18.4)$ & 0.491 \\
\hline Anti-hypertensives & $7,572(90.7)$ & $6,445(89.8)$ & 1,127 (96.4) & $<0.001$ \\
\hline ACE inhibitor & $783(9.4)$ & $634(8.8)$ & $149(12.8)$ & $<0.001$ \\
\hline СCB & $3,805(45.6)$ & $3,214(44.8)$ & $591(50.6)$ & $<0.001$ \\
\hline Diuretic & 2,494 (29.9) & 1,912 (26.6) & $582(49.8)$ & $<0.001$ \\
\hline Beta-blocker & $5,374(64.4)$ & $4,588(63.9)$ & $786(67.2)$ & 0.027 \\
\hline Alpha-blocker & $150(1.8)$ & $128(1.8)$ & $22(1.9)$ & 0.813 \\
\hline NOAC & $58(0.7)$ & $9(0.1)$ & $49(4.2)$ & $<0.001$ \\
\hline
\end{tabular}

AF, atrial fibrillation; MI, myocardial infarction; PAD, peripheral artery disease; COPD, chronic obstructive pulmonary disease; ESRD, end-stage renal disease; SBP, systolic blood pressure; DBP, diastolic blood pressure; LDL-C, low-density lipoprotein cholesterol; HDL-C, high-density lipoprotein cholesterol; $\mathrm{CrCl}$, creatinine clearance; ALT, alanine transaminase; AST, aspartate transaminase; GGT, gamma-glutamyltransferase; ACE, angiotensin-converting-enzyme; ARB, angiotensin II receptor blocker; CCB, calcium channel blocker; NOAC, non-vitamin $\mathrm{K}$ antagonist oral anticoagulants.

\section{Impact of AF on the risk of stroke and death in HCM patients}

During a mean follow-up of $2.5 \pm 1.5$ years, ischemic stroke occurred in 484 (6.7\%) of non-AF patients and $152(13.0 \%)$ of $\mathrm{AF}$ patients. The incidence rate of stroke was 2.69 and 5.87 per 100 person-years in non-AF and AF subjects, respectively. Patients with $\mathrm{AF}$ showed a $60 \%$ increase in risk for stroke compared to those without $\mathrm{AF}$ after multivariable analysis adjusting for confounding variables including anticoagulation (HR 1.60, 95\% CI: 1.26-2.04) (Table 2).

Of note, the impact of $\mathrm{AF}$ on increased risk for stroke was even stronger in HCM patients who had fewer risk factors, represented by lower $\mathrm{CHA}_{2} \mathrm{DS}_{2}$-VASc scores (Figure 2A). HCM patients with $\mathrm{AF}$ showed high incidence rates of stroke even with few risk factors: 3.37 and 2.94 per 100 person-years for $\mathrm{CHA}_{2} \mathrm{DS}_{2}-\mathrm{VASc}=0$ and 1 , respectively. Patients who had $\mathrm{AF}$ and no other risk factors $\left(\mathrm{CHA}_{2} \mathrm{DS}_{2}-\right.$ VASc $=0$ ) showed more than 4-fold increased risk for stroke compared to those without $\mathrm{AF}$ and $\mathrm{CHA}_{2} \mathrm{DS}_{2}-\mathrm{VASc}=0$. Meanwhile, in HCM patients with multiple risk factors $\left(\mathrm{CHA}_{2} \mathrm{DS}_{2}\right.$-VASc score $\left.\geq 4\right)$, the increase in $\mathrm{AF}$-associated stroke risk was not significant.

On the other hand, during a mean follow-up of
$2.6 \pm 1.5$ years, all-cause death occurred in 391 (5.4\%) of non-AF patients and 127 (10.9\%) of AF patients. Mortality rate was 2.06 and 4.44 per 100 person-years in non-AF and AF patients, respectively. HCM patients with AF showed a $49 \%$ increase in risk for death compared to those without $\mathrm{AF}$ in multivariable analysis (Table 2). AF-associated risk for death was consistently increased in HCM patients throughout $\mathrm{CHA}_{2} \mathrm{DS}_{2}$-VASc scores (Figure $2 B$ ).

\section{Performance of $C H A_{2} D S_{2}-V A S c$ score for predicting stroke in HCM patients not on anticoagulation}

The incidence rates and hazard ratios for developing ischemic stroke according to $\mathrm{CHA}_{2} \mathrm{DS}_{2}$-VASc scores in HCM patients not on anticoagulation are shown in Figure 3. $\mathrm{HCM}$ patients with $\mathrm{AF}$ and low $\mathrm{CHA}_{2} \mathrm{DS}_{2}-$ VASc scores showed high incidences of stroke: 2.50 and 2.75 per 100 person-years for $\mathrm{CHA}_{2} \mathrm{DS}_{2}$-VASc $=0$ and 1 , respectively. Incidence rates of stroke generally increased with higher $\mathrm{CHA}_{2} \mathrm{DS}_{2}$-VASc scores, and patients with very high $\mathrm{CHA}_{2} \mathrm{DS}_{2}$-VASc scores $(\geq 6)$ had markedly elevated incidence rates of stroke. Due to the high incidence of stroke in HCM patients with low $\mathrm{CHA}_{2} \mathrm{DS}_{2}$-VASc scores, there were no statistically significant differences in risk of 
Table 2 Impact of atrial fibrillation (AF) on the risk of stroke and mortality in hypertrophic cardiomyopathy patients

\begin{tabular}{|c|c|c|c|c|c|c|}
\hline & \multicolumn{2}{|c|}{ Non-AF $(n=7,180)$ (reference) } & \multicolumn{4}{|c|}{$\mathrm{AF}(\mathrm{n}=1,169)$} \\
\hline Stroke & 484 & 2.69 & 152 & 5.87 & $2.16(1.80-2.59)$ & $1.60(1.26-2.04)$ \\
\hline All-cause death & 391 & 2.06 & 127 & 4.44 & $2.22(1.82-2.71)$ & $1.49(1.14-1.95)$ \\
\hline
\end{tabular}

${ }^{\dagger}$ Incidence rate: per 100 person-years. ${ }^{\ddagger}$ Adjusted for age, sex, comorbidities (hypertension, diabetes mellitus, dyslipidemia, heart failure, prior myocardial infarction, peripheral artery disease, chronic obstructive pulmonary disease, end-stage renal disease), body mass index, creatinine clearance, smoking, drinking, exercise, presence of oral anticoagulation. HR, hazard ratio; $\mathrm{Cl}$, confidence interval.

A Ischemic stroke

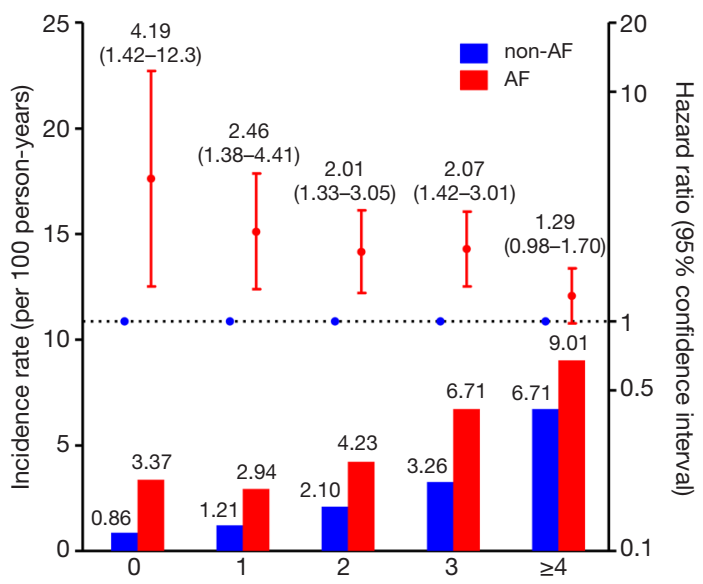

B All-cause death

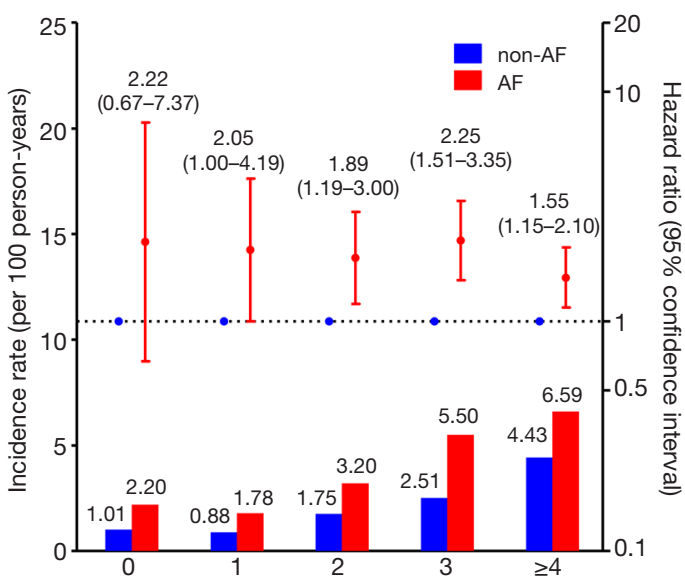

Figure 2 Impact of atrial fibrillation (AF) on the risk of (A) stroke and (B) mortality in hypertrophic cardiomyopathy patients, stratified by $\mathrm{CHA}_{2} \mathrm{DS}_{2}$-VASc scores. Incidence rates (per 100 person-years) of stroke for each $\mathrm{CHA}_{2} \mathrm{DS}_{2}$-VASc scores are shown in bar graphs at the bottom with scales on the left. Hazard ratios with $95 \%$ confidence intervals representing the risk of stroke for each $\mathrm{CHA}_{2} \mathrm{DS}_{2}-\mathrm{VASc}_{\mathrm{Scores}}$ are shown in line graphs with scales (base 10 logarithmic) on the right, with a dotted line at 1.

stroke among patients with $\mathrm{CHA}_{2} \mathrm{DS}_{2}$-VASc scores $1-5$ compared to those with $\mathrm{CHA}_{2} \mathrm{DS}_{2}$-VASc $=0$, though there was a trend for linear increase in stroke risk in proportion to $\mathrm{CHA}_{2} \mathrm{DS}_{2}$-VASc scores (Figure $3 A$ ). The risk of stroke was significantly increased only at very high $\mathrm{CHA}_{2} \mathrm{DS}_{2}-$ VASc scores $(\geq 6)$ in multivariable adjusted Cox regression analysis. Analyses in total AF patients showed similar results, with high incidence rates of stroke at low $\mathrm{CHA}_{2} \mathrm{DS}_{2}-$ VASc scores, and no significant difference in stroke risk at $\mathrm{CHA}_{2} \mathrm{DS}_{2}-\mathrm{VASc}<6$ (Table S2). The $\mathrm{CHA}_{2} \mathrm{DS}_{2}$-VASc scoring system showed poor discrimination of stroke risk in HCM patients with AF (AUC 0.591, 95\% CI: 0.501-0.673) at three years of follow-up (Figure $3 B$ ).

On the other hand, incidence rates and hazard ratios of developing ischemic stroke showed an increase with higher $\mathrm{CHA}_{2} \mathrm{DS}_{2}$-VASc scores in HCM patients without
AF (Figure $\mathrm{S} 1$ ). The $\mathrm{CHA}_{2} \mathrm{DS}_{2}$-VASc scoring system showed better discrimination of stroke risk in HCM patients without AF at three years of follow-up (AUC 0.717, 95\% CI: 0.691-0.742) compared to those with AF (P for comparison of independent ROC curves $=0.002$ ).

\section{Risk factors for stroke in HCM patients with and without AF}

In AF-free HCM patients, traditional risk factors such as age, female sex, hypertension, diabetes mellitus, dyslipidemia, heart failure, peripheral artery disease, and chronic obstructive pulmonary disease were associated with incident stroke in univariable analysis, and of these, age and peripheral artery disease remained significantly associated with increased risk for stroke in multivariable analysis. 

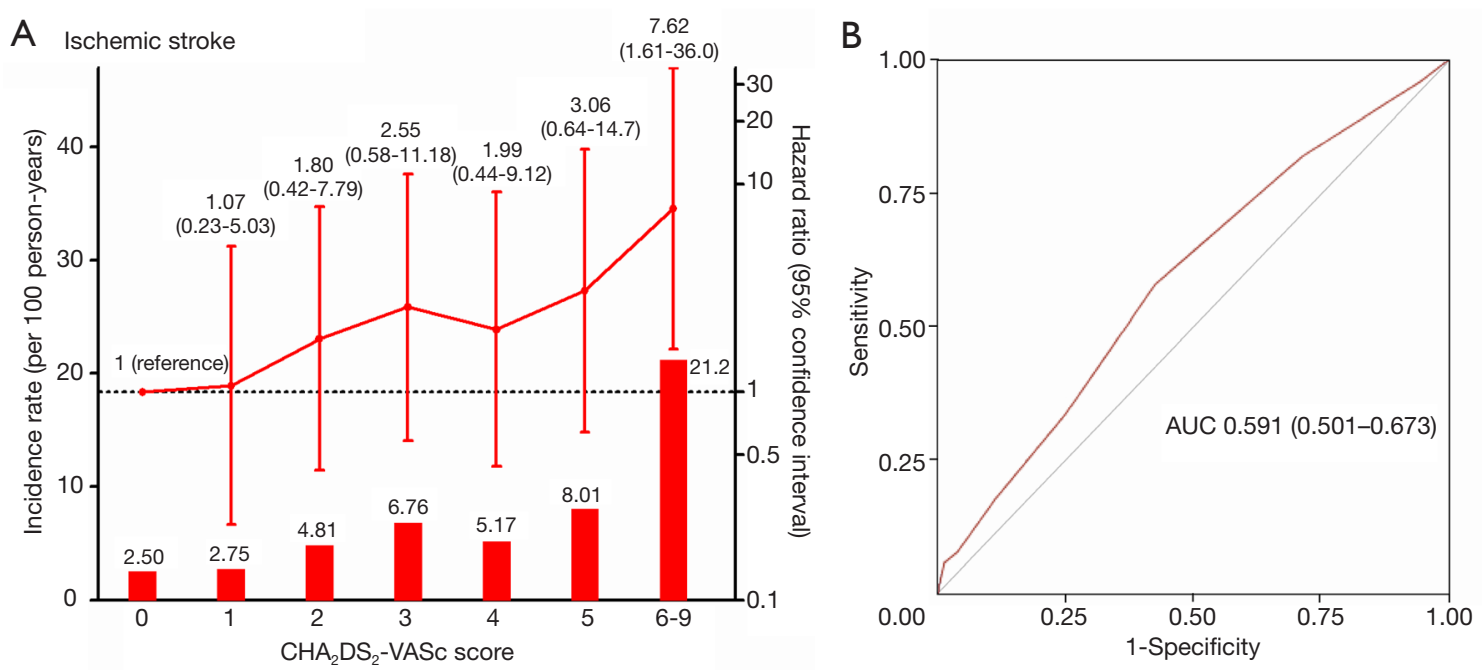

Figure 3 Performance of the $\mathrm{CHA}^{2} \mathrm{DS}^{2}$-VASc score for predicting stroke in hypertrophic cardiomyopathy patients with atrial fibrillation and without anticoagulation. (A) Incidence rates (per 100 person-years) of stroke for each CHA2DS2-VASc scores are shown in bar graphs at the bottom with scales on the left. Hazard ratios with 95\% confidence intervals representing the risk of stroke for each CHA2DS2-VASc scores are shown in line graphs with scales (base 10 logarithmic) on the right, with a dotted line at 1 . (B) ROC curve for the prediction of stroke at 3 years of follow-up.

Meanwhile, in HCM patients with AF, age and heart failure in univariable and multivariable analysis were associated with incident stroke (Figure 4, Table S3).

Of various health checkup variables (Figure 4, Table S4), low creatinine clearance $(<60 \mathrm{~mL} / \mathrm{min})$, high AST and GGT [highest quartile (Q); Q4] were associated with higher stroke risk, while high body mass index $(\geq 25)$ was associated with lower stroke risk in AF-free HCM patients. Meanwhile, in HCM patients with AF, high blood pressure $(\geq 160 / 100 \mathrm{mmHg}$ ) and GGT (Q4) were associated with incident stroke. Quartile ranges are shown in Table S5.

\section{Discussion}

We found that in a nationwide cohort of patients with HCM, $\mathrm{AF}$ was common (14\%), and independently associated with approximately $60 \%$ and $50 \%$ increased risk for stroke and mortality, respectively, in multivariable analysis adjusting for confounding variables such as comorbidities, lifestyle, body mass index, kidney function, and anticoagulation. In particular, the $\mathrm{AF}$-associated increase in risk of stroke was more prominent in HCM patients with fewer risk factors. The $\mathrm{CHA}_{2} \mathrm{DS}_{2}-\mathrm{VASc}$ scoring system showed a poor performance at stroke prediction in HCM patients with AF, and those with low scores showed high incidence rates of stroke. Traditional risk factors were not always associated with stroke in $\mathrm{HCM}$ patients with AF; age, heart failure, high blood pressure and GGT were the strongest predictors of stroke in this population.

\section{Clinical implications of AF in HCM in the contemporary management era}

The evolution of contemporary therapeutic strategies have substantially improved the clinical course and longevity of HCM patients, achieving a low mortality rate comparable to that of the general population (5). In studies of HCM patients followed up before $2000(1,2,20)$, and also in more contemporary studies spanning periods before and after $2000(3,4), \mathrm{AF}$ was associated with a substantially higher risk of stroke and death, especially stroke- and heart failurerelated mortality. Meanwhile, a study by Rowin et al. from a single-institution referral HCM population using standardized management with a high rate of aggressive procedures, evaluated between 2004 and 2014, found that AF impairs the quality of life, but does not increase all-cause or HCM-related death or progression of heart failure (6), which was attributed to improved contemporary management strategies, including effective anticoagulation to prevent embolic stroke. However, most of the data are from Western countries, and the clinical implications of AF in contemporary Asian HCM patients are lacking. 


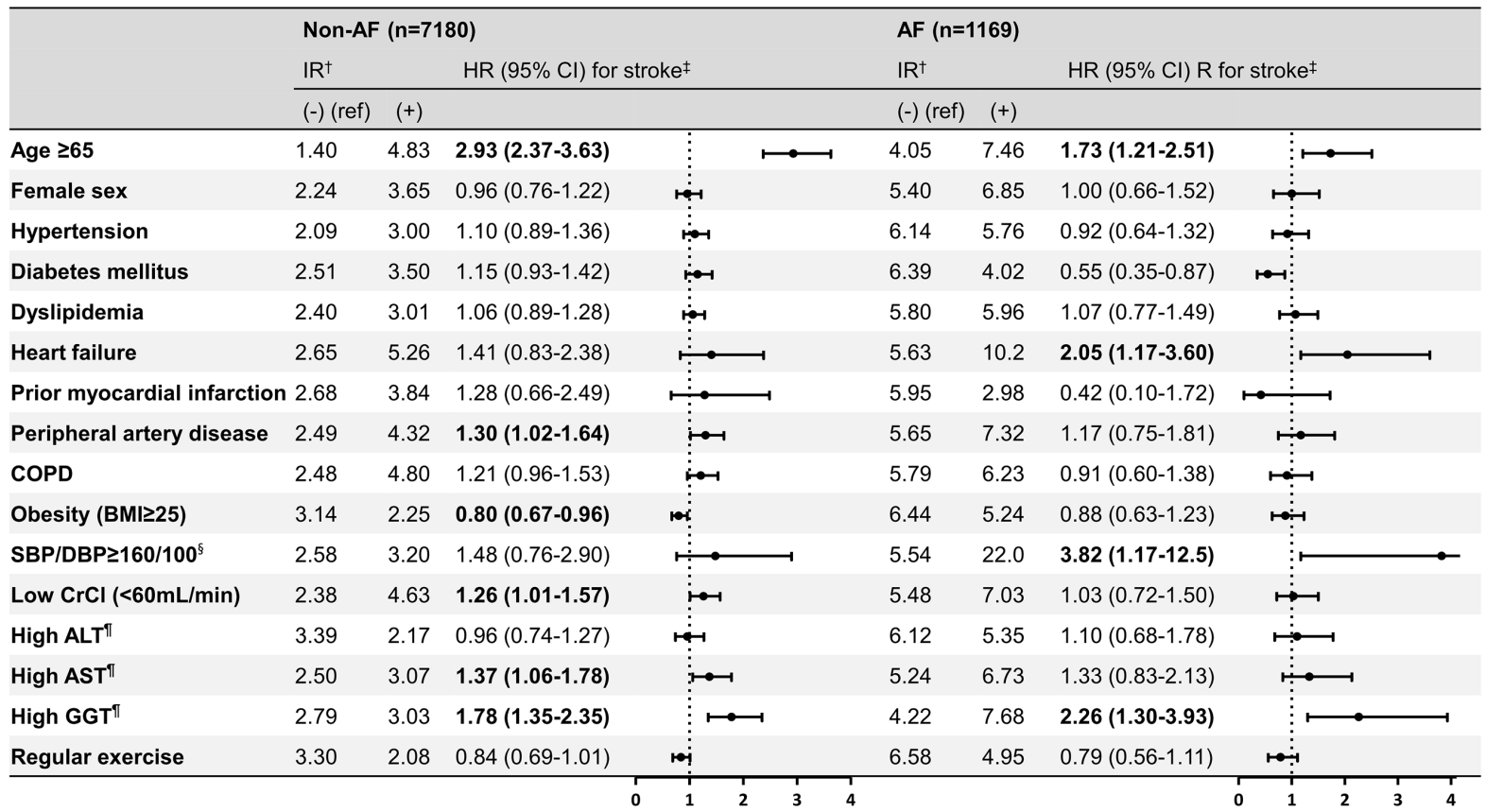

Figure 4 Risk factors for stroke in hypertrophic cardiomyopathy patients with and without AF. ${ }^{\dagger}$ Incidence rate: per 100 person-years. ${ }^{\ddagger}$ Adjusted for age, comorbidities (hypertension, diabetes mellitus, dyslipidemia, heart failure, prior myocardial infarction, peripheral artery disease, COPD, ESRD), BMI, CrCl, smoking, drinking, exercise, presence of oral anticoagulation. ${ }^{\$} v s$. SBP/DBP < $120 / 80$ (reference). ${ }^{\mathbb{I}} \mathrm{Q} 4$ (4th quartile) vs. Q1 (1st quartile; reference). AF, atrial fibrillation; HR, hazard ratio; CI, confidence interval; COPD, chronic obstructive pulmonary disease; ESRD, end-stage renal disease; BMI, body mass index; $\mathrm{CrCl}$, creatinine clearance; ALT, alanine transaminase; AST, aspartate transaminase; GGT, gamma-glutamyltransferase.

In our study, $\mathrm{AF}$ remains a significant contributor to morbidity and mortality in a nationwide HCM cohort from 2010 to 2016. Our study population included all HCM patients from a nationwide health insurance database, and thus the study population is free from referral bias and is more community-based, which is different from the above studies performed with tertiary referral population. Anticoagulation rates were sub-optimal in the present study, as only $51.7 \%$ of $\mathrm{HCM}$ patients with $\mathrm{AF}$ were anticoagulated; however, this proportion is not so different from that of other cohort studies involving $\mathrm{HCM}$ patients with $\mathrm{AF}$, where anticoagulation rates ranged from $41 \%$ to $78 \%(3,6)$. In this referral bias-free setting, AF was still associated with higher stroke and mortality rates even after multivariable adjustment including the presence of anticoagulation, especially in HCM patients with fewer risk factors.

\section{Prediction of stroke in HCM patients}

The $\mathrm{CHA}_{2} \mathrm{DS}_{2}$-VASc scoring system showed poor discrimination of stroke risk, which only increased significantly at very high scores $(\geq 6)$, in HCM patients with AF. A main reason behind its poor performance was that those with low $\mathrm{CHA}_{2} \mathrm{DS}_{2}$-VASc scores, even 0 or 1 , showed high stroke rates. The presence of HCM itself conferred a steep increase in risk of stroke, which attenuated further increase in stroke risk by addition of other risk factors, and thus led to the poor performance of the $\mathrm{CHA}_{2} \mathrm{DS}_{2}$-VASc score. This is consistent with previous studies showing high incidence rates of stroke in $\mathrm{HCM}$ patients with $\mathrm{AF}$ and low $\mathrm{CHA}_{2} \mathrm{DS}_{2}$-VASc scores $(21,22)$. These results support that the $\mathrm{CHA}_{2} \mathrm{DS}_{2}$-VASc score should not be used in HCM patients with $\mathrm{AF}$ in the decision to start anticoagulation, and also corroborate current guidelines recommending that HCM patients with AF should be anticoagulated regardless of $\mathrm{CHA}_{2} \mathrm{DS}_{2}$-VASc scores $(8,9)$. The hemodynamics of HCM including diastolic dysfunction, left atrial dilatation, and outflow obstruction may provide a potent substrate for stagnancy, turbulence and thrombus formation. This may be synergized by the development of AF, which could explain 
the steep rise in stroke rates even at low $\mathrm{CHA}_{2} \mathrm{DS}_{2}$-VASc scores in HCM patients with AF. Interestingly, even HCM patients without $\mathrm{AF}$ had increased incidence of stroke, and the $\mathrm{CHA}_{2} \mathrm{DS}_{2}$-VASc score showed good discrimination of stroke risk in this population. The incidence of stroke in $\mathrm{HCM}$ patients without AF was similar to that of the general population with AF derived from the same database (23) or from previous studies (24), again suggesting that HCM per se increases the risk for stroke, perhaps approximately as much as $\mathrm{AF}$ in the general population. In particular, $\mathrm{HCM}$ patients without $\mathrm{AF}$ at baseline but $\mathrm{CHA}_{2} \mathrm{DS}_{2}$-VASc $\geq 1$ showed $>1 \%$ yearly incidence of ischemic stroke, and further studies exploring whether anticoagulation could be beneficial in this group would be of interest.

Considering the suboptimal performance of the $\mathrm{CHA}_{2} \mathrm{DS}_{2}$-VASc score in $\mathrm{HCM}$ patients with AF, we explored the potential predictors of stroke in this population. While the current study may not have sufficient power to be conclusive, we found that age, heart failure, high blood pressure and GGT were the strongest predictors of stroke in HCM patients with $\mathrm{AF}$, while other traditional risk factors were not. GGT is expressed in the cerebrovascular endothelium as well as in the liver and kidney, and besides being a proxy marker of alcohol consumption, also plays a role in oxidative stress and the pathogenesis of atherosclerosis. Previous studies have also reported that GGT is associated with cardiovascular disease including ischemic stroke, metabolic syndrome, and $\mathrm{AF}$, independently of alcohol intake or liver disease (25-28). We here first demonstrated the possibility of GGT as a biomarker predicting stroke in HCM patients with and without AF.

\section{Limitations}

Several limitations need to be mentioned. First, we excluded HCM patients without health examination results from the prevalent HCM population (38\%, 6,436/16,886), and thus the study population may not reflect the full spectrum of the HCM population. Subjects who did not undergo routine health examinations may show different characteristics from those who did; they may be 'sicker' and thus prone to skip check-ups due to frequent hospital visits, or socially isolated without convenient access to healthcare, or poorly adherent. In this respect, selection bias cannot be completely excluded. Second, patient groups with and without AF show different characteristics, and despite multiple adjustments, residual confounding may exist. Third, we did not include patients with prior stroke for accurate assessment of clinical outcomes in the claims database, which may lead to selection bias. Fourth, although the presence or absence of anticoagulation was adjusted for in multivariate analysis, information on the effectiveness of anticoagulation, especially in warfarin users, could not be collected. Finally, detailed echocardiographic information was unavailable in the claims database, which would have strengthened our results.

\section{Conclusions}

In a nationwide contemporary population of HCM patients with $\mathrm{AF}$ in Korea, AF was independently associated with approximately $60 \%$ and $50 \%$ increased risk for stroke and mortality, respectively. AF-associated increase in stroke was especially marked in patients with fewer risk factors. The $\mathrm{CHA}_{2} \mathrm{DS}_{2}$-VASc score showed poor discrimination of stroke risk in HCM patients with AF, mainly due to the high incidence of stroke even at low $\mathrm{CHA}_{2} \mathrm{DS}_{2}$-VASc scores, and should not be used in deciding whether to start anticoagulation in patients with $\mathrm{HCM}$ and AF. Age, heart failure, high blood pressure, and GGT emerged as the strongest predictors of stroke in patients with $\mathrm{HCM}$ and $\mathrm{AF}$.

\section{Acknowledgments}

Funding: This work was supported by the 2019 Hanmi research fund (grant number 0620192190).

\section{Footnote}

Reporting Checklist: The authors have completed the STROBE reporting checklist. Available at http://dx.doi. org/10.21037/atm-20-1817

Peer Review File: Available at http://dx.doi.org/10.21037/ atm-20-1817

Conflicts of Interest: All authors have completed the ICMJE uniform disclosure form (available at http://dx.doi. org/10.21037/atm-20-1817). HKK reports grants from 2019 Hanmi research fund during the conduct of the study; grants from Actelion, Handok, Dae-Woong, Hanmi, Norvatis, JW phamaceuticals, Samjin, and DongA, outside the submitted work. The other authors have no conflicts of interest to declare.

Ethical Statement: The authors are accountable for all 
aspects of the work in ensuring that questions related to the accuracy or integrity of any part of the work are appropriately investigated and resolved. The study was conducted in accordance with the Declaration of Helsinki (as revised in 2013). This study was exempt from review by the Seoul National University Hospital Institutional Review Board (E-1806-016-949) due to the anonymized nature of the data and individual consent was waived.

Open Access Statement: This is an Open Access article distributed in accordance with the Creative Commons Attribution-NonCommercial-NoDerivs 4.0 International License (CC BY-NC-ND 4.0), which permits the noncommercial replication and distribution of the article with the strict proviso that no changes or edits are made and the original work is properly cited (including links to both the formal publication through the relevant DOI and the license). See: https://creativecommons.org/licenses/by-nc-nd/4.0/.

\section{References}

1. Olivotto I, Cecchi F, Casey SA, et al. Impact of atrial fibrillation on the clinical course of hypertrophic cardiomyopathy. Circulation 2001;104:2517-24.

2. Maron BJ, Olivotto I, Bellone P, et al. Clinical profile of stroke in 900 patients with hypertrophic cardiomyopathy. $\mathrm{J}$ Am Coll Cardiol 2002;39:301-7.

3. Siontis KC, Geske JB, Ong K, et al. Atrial fibrillation in hypertrophic cardiomyopathy: prevalence, clinical correlations, and mortality in a large high-risk population. J Am Heart Assoc 2014;3:e01002.

4. Guttmann OP, Pavlou M, O'Mahony C, et al. Predictors of atrial fibrillation in hypertrophic cardiomyopathy. Heart 2017;103:672-8.

5. Maron BJ, Rowin EJ, Casey SA, et al. Hypertrophic Cardiomyopathy in Adulthood Associated With Low Cardiovascular Mortality With Contemporary Management Strategies. J Am Coll Cardiol 2015;65:1915-28.

6. Rowin EJ, Hausvater A, Link MS, et al. Clinical Profile and Consequences of Atrial Fibrillation in Hypertrophic Cardiomyopathy. Circulation 2017;136:2420-36.

7. Guttmann OP, Pavlou M, O'Mahony C, et al. Prediction of thrombo-embolic risk in patients with hypertrophic cardiomyopathy (HCM Risk-CVA). Eur J Heart Fail 2015;17:837-45.

8. Gersh BJ, Maron BJ, Bonow RO, et al. 2011 ACCF/ AHA guideline for the diagnosis and treatment of hypertrophic cardiomyopathy: a report of the American College of Cardiology Foundation/American Heart Association Task Force on Practice Guidelines. Circulation 2011;124:e783-831.

9. Authors/Task Force members, Elliott PM, Anastasakis A, et al. 2014 ESC Guidelines on diagnosis and management of hypertrophic cardiomyopathy: the Task Force for the Diagnosis and Management of Hypertrophic Cardiomyopathy of the European Society of Cardiology (ESC). Eur Heart J 2014;35:2733-79.

10. Lee HJ, Choi EK, Lee SH, et al. Risk of ischemic stroke in metabolically healthy obesity: A nationwide populationbased study. PLoS One 2018;13:e0195210.

11. Lee SR, Lee HJ, Choi EK, et al. Direct Oral Anticoagulants in Patients With Atrial Fibrillation and Liver Disease. J Am Coll Cardiol 2019;73:3295-308.

12. Lee HJ, Kim HK, Jung JH, et al. Novel Oral Anticoagulants for Primary Stroke Prevention in Hypertrophic Cardiomyopathy Patients With Atrial Fibrillation. Stroke 2019;50:2582-6.

13. Choi YJ, Choi EK, Han KD, et al. Temporal trends of the prevalence and incidence of atrial fibrillation and stroke among Asian patients with hypertrophic cardiomyopathy: A nationwide population-based study. Int J Cardiol 2018;273:130-5.

14. Kim TH, Yang PS, Uhm JS, et al. CHA2DS2-VASc Score (Congestive Heart Failure, Hypertension, Age $>/=75$ [Doubled], Diabetes Mellitus, Prior Stroke or Transient Ischemic Attack [Doubled], Vascular Disease, Age 6574, Female) for Stroke in Asian Patients With Atrial Fibrillation: A Korean Nationwide Sample Cohort Study. Stroke 2017;48:1524-30.

15. Lip GY, Nieuwlaat R, Pisters R, et al. Refining clinical risk stratification for predicting stroke and thromboembolism in atrial fibrillation using a novel risk factor-based approach: the euro heart survey on atrial fibrillation. Chest 2010;137:263-72.

16. Ntaios G, Lip GY, Makaritsis K, et al. CHADS(2), CHA(2)S(2)DS(2)-VASc, and long-term stroke outcome in patients without atrial fibrillation. Neurology 2013;80:1009-17.

17. Mitchell LB, Southern DA, Galbraith D, et al. Prediction of stroke or TIA in patients without atrial fibrillation using CHADS2 and CHA2DS2-VASc scores. Heart 2014;100:1524-30.

18. Parsons C, Patel SI, Cha S, et al. CHA2DS2-VASc Score: A Predictor of Thromboembolic Events and Mortality in Patients With an Implantable Monitoring Device Without 
Atrial Fibrillation. Mayo Clin Proc 2017;92:360-9.

19. DeLong ER, DeLong DM, Clarke-Pearson DL. Comparing the areas under two or more correlated receiver operating characteristic curves: a nonparametric approach. Biometrics 1988;44:837-45.

20. Guttmann OP, Rahman MS, O'Mahony C, et al. Atrial fibrillation and thromboembolism in patients with hypertrophic cardiomyopathy: systematic review. Heart 2014;100:465-72.

21. Tsuda T, Hayashi K, Fujino N, et al. Effect of hypertrophic cardiomyopathy on the prediction of thromboembolism in patients with nonvalvular atrial fibrillation. Heart Rhythm 2019;16:829-37.

22. Jung H, Yang PS, Sung JH, et al. Hypertrophic Cardiomyopathy in Patients with Atrial Fibrillation: Prevalence and Associated Stroke Risks in a Nationwide Cohort Study. Thromb Haemost 2019;119:285-93.

23. Kang SH, Choi EK, Han KD, et al. Risk of Ischemic Stroke in Patients With Non-Valvular Atrial Fibrillation Not Receiving Oral Anticoagulants- Korean Nationwide Population-Based Study. Circ J 2017;81:1158-64.

24. European Heart Rhythm Association, European

Cite this article as: Lee $\mathrm{HJ}, \mathrm{Kim} H K, \operatorname{Kim} M$, Moon I, Lee H, Kim BS, Han KD, Park JB, Hwang IC, Yoon YE, Kim YJ, Cho GY, Ommen SR. Clinical impact of atrial fibrillation in a nationwide cohort of hypertrophic cardiomyopathy patients. Ann Transl Med 2020;8(21):1386. doi: 10.21037/atm-20-1817
Association for Cardio-Thoracic Surgery, Camm AJ, et al. Guidelines for the management of atrial fibrillation: the Task Force for the Management of Atrial Fibrillation of the European Society of Cardiology (ESC). Eur Heart J 2010;31:2369-429.

25. Ruttmann E, Brant LJ, Concin H, et al. Gammaglutamyltransferase as a risk factor for cardiovascular disease mortality: an epidemiological investigation in a cohort of 163,944 Austrian adults. Circulation 2005;112:2130-7.

26. Fraser A, Harris R, Sattar N, et al. Gammaglutamyltransferase is associated with incident vascular events independently of alcohol intake: analysis of the British Women's Heart and Health Study and MetaAnalysis. Arterioscler Thromb Vasc Biol 2007;27:2729-35.

27. Kunutsor SK, Apekey TA, Khan H. Liver enzymes and risk of cardiovascular disease in the general population: a meta-analysis of prospective cohort studies. Atherosclerosis 2014;236:7-17.

28. Yang W, Kim CK, Kim DY, et al. Gamma-glutamyl transferase predicts future stroke: A Korean nationwide study. Ann Neurol 2018;83:375-86. 